\title{
Ações e políticas públicas nos manguezais para a preservação dos serviços ambientais
}

\author{
Public actions and policies in mangroves for the preservation of environmental \\ Acciones y políticas públicas en manglares para la preservación de los servicios ambientales
}

Recebido: 15/11/2021 | Revisado: 20/11/2021 | Aceito: 25/11/2021 | Publicado: 29/11/2021

\author{
Elton Januario Silva \\ ORCID: https://orcid.org/0000-0003-4526-0554 \\ Universidade Federal de Campina Grande, Brasil \\ E-mail eltonjsilva@outlook.com \\ Isabel Lausanne Fontgalland \\ ORCID https://orcid.org/0000-0002-0087-2840 \\ Universidade Federal de Campina Grande, Brasil \\ E-mail: isabelfontgalland@gmail.com
}

\begin{abstract}
Resumo
Os ecossistemas são as bases da ecologia. A relação entre eles e o homem se dá através do uso dos seus serviços ambientais, o que finda na quebra da sua resiliência ou depleção. Neste aspecto, estão inseridos os manguezais, extremamente importantes como fonte de renda para comunidades em seu entorno. O objetivo deste trabalho foi o de discutir ações do seu uso e conservação e se há integração com políticas públicas no Brasil e no Mundo. A pesquisa realizada foi exploratória. Os manguezais estão presentes entre as áreas tropicais, principalmente entre as latitudes $30^{\circ}$ $\mathrm{N}$ e $30^{\circ} \mathrm{S}$, tendo o Brasil aproximadamente $13.989 \mathrm{~km}^{2}$ desta feição em sua costa, com $87 \%$ presente no interior de Unidades de Conservação. Percebeu-se que, no Brasil, a lei ainda está se adequando a necessidade da proteção destes ecossistemas, mudando os conceitos presentes em suas letras para preservação com o passar dos anos. Nos Estados Unidos, as leis da Flórida mostram foco no desenvolvimento sustentável. A valoração dos manguezais possui importância no estabelecimento de políticas públicas e investimentos, beneficiando comunidades envolvidas com eles. A convenção Ramsar estabelece formas de uso sustentável de zonas úmidas em vários países. Esta convenção não faz distinção de áreas, e no Brasil as leis o emulam, onde o manguezal compartilha as mesmas considerações que outras feições. Percebeu-se que legislação brasileira para os manguezais não possui especificidades que tratem do seu uso sustentável, mas que os projetos criados nestas áreas possuem resultados bastante importantes e satisfatórios.
\end{abstract}

Palavras-chave: Ecossistema manguezal; Políticas públicas; Serviços ambientais.

\begin{abstract}
Ecosystems are the foundation of ecology. The relationship between them and man takes place through the use of their environmental services, which ends up in the breakdown of their resilience or depletion. In this aspect, mangroves are included, which are extremely important as a source of income for surrounding communities. The objective of this work was to discuss actions of its use and conservation and if there is integration with public policies in Brazil and in the world. The research carried out was exploratory. Mangroves are present among tropical areas, mainly between latitudes $30^{\circ} \mathrm{N}$ and $30^{\circ} \mathrm{S}$, with Brazil having approximately $13,989 \mathrm{~km}^{2}$ of this feature on its coast, with $87 \%$ present in the interior of Conservation Units. It was noticed that, in Brazil, the law is still adapting to the need to protect these ecosystems, changing the concepts present in its letters for preservation over the years. In the United States, Florida laws show a focus on sustainable development. The valuation of mangroves is important in establishing public policies and investments, benefiting communities involved with them. The Ramsar convention establishes ways of sustainable use of wetlands in several countries. This convention makes no distinction between areas, and in Brazil the laws emulate it, where mangroves share the same considerations as other features. It was noticed that Brazilian legislation for mangroves does not have specificities that deal with their sustainable use, but that the projects created in these areas have very important and satisfactory results.
\end{abstract}

Keywords: Mangrove ecosystem; Public policy; Environmental services.

\section{Resumen}

Los ecosistemas son la base de la ecología. La relación entre ellos y el hombre se da a través del uso de sus servicios ambientales, que termina en la ruptura de su resiliencia o agotamiento. En este aspecto se incluyen los manglares, los cuales son de suma importancia como fuente de ingresos para las comunidades aledañas. El objetivo de este trabajo fue discutir acciones de su uso y conservación y si hay integración con las políticas públicas en Brasil y en el mundo. La investigación realizada fue exploratoria. Los manglares están presentes entre las áreas tropicales, principalmente entre las latitudes $30^{\circ} \mathrm{N}$ y $30^{\circ} \mathrm{S}$, teniendo Brasil aproximadamente $13,989 \mathrm{~km}^{2}$ de esta característica en su costa, con $87 \%$ presente en el interior de las Unidades de Conservación. Se notó que, en Brasil, la ley aún se está adaptando a la necesidad de proteger estos ecosistemas, cambiando los conceptos presentes en sus cartas para la preservación a lo 
largo de los años. En los Estados Unidos, las leyes de Florida muestran un enfoque en el desarrollo sostenible. La valoración de los manglares es importante en el establecimiento de políticas públicas e inversiones, beneficiando a las comunidades involucradas con ellos. La convención Ramsar establece formas de uso sostenible de los humedales en varios países. Esta convención no hace distinción entre áreas, y en Brasil las leyes la emulan, donde los manglares comparten las mismas consideraciones que otras características. Se notó que la legislación brasileña para los manglares no tiene especificidades que se ocupen de su uso sostenible, pero que los proyectos creados en estas áreas tienen resultados muy importantes y satisfactorios.

Palabras clave: Ecossistema de manglar; Políticas públicas; Servicios ambientales.

\section{Introdução}

Os ecossistemas são as essências da composição dos biomas. São definidos como unidades básicas funcionais da ecologia, abarcando os fatores bióticos e abióticos que compõem a estrutura de uma paisagem e que são necessários para a sobrevivência da vida na Terra.

A respeito desta classificação, ela insere o conceito de ecossistema na base fundamental organizacional das estruturas vivas, sendo estas conscientes, sencientes ou mais simples. Em se tratando dos conscientes, objetivamente o homem, que os modifica de acordo com a sua necessidade e os utiliza como alicerce de todas as atividades necessárias para o desenvolvimento da sociedade.

A interrelação entre os seres vivos e o meio ambiente criam condições para o surgimento dos serviços denominados ecossistêmicos, originando os ecossistemas em si. Já a utilização dos ecossistemas pelo homem é realizada através dos seus serviços ambientais, baseando-se no uso de suas benesses, alteradas ou não anteriormente pela influência antrópica.

A exploração histórica dos serviços ambientais para este desenvolvimento culmina ao atingirem o limite das resiliências dos ecossistemas dos quais eles fazem parte, o que faz com que a recuperação das áreas exploradas se torna algo complexo devido à perda de suas características iniciais. A necessidade do uso sustentável dos serviços ambientais se converteu em algo cada vez maior em função da sua exploração pelas comunidades e indústrias que os permeiam.

Dentre os ecossistemas que sofrem mais pressão antrópica evidente estão notoriamente os manguezais, formados pelas plantas lenhosas presentes na interface existente entre a terra e o mar e em latitudes tropicais e subtropicais, associadas a micróbios, fungos, plantas, animais e fatores abióticos.

O ecossistema manguezal constitui a mais importante fonte de renda para as populações que os margeiam, através dos seus serviços ambientais. Possui uma grande importância socioambiental, pois diversas espécies marinhas e aves o utilizam como área de reprodução e alimentação. Pescadores e catadores de crustáceos também fazem o uso dessas áreas para retirar seu sustento. No entanto, as intensas intervenções antrópicas têm influenciado as funcionalidades e a prestação dos seus serviços ecossistêmicos, tornando-o um dos mais ameaçados.

Diante desta importância, no Brasil e no mundo foram realizadas iniciativas para coibir o uso indiscriminado do manguezal, criando defesas para os sistemas ecossistêmicos e ambientais, tendo como base políticas públicas, a legislação das áreas e valoração econômica. O objetivo deste trabalho é realizar uma síntese de ações de uso e conservação do ecossistema manguezal e a sua integração às políticas públicas ambientais vigentes, no Brasil e no mundo, e como, através destas ações, se realiza a conscientização de sua importância.

\section{Metodologia}

O estudo foi realizado através de pesquisa exploratória, tendo como fonte de investigação revistas, sites da internet e artigos acadêmicos, além de leis do Brasil e do mundo que tratam, sendo auxiliada por ferramentas de indexação de referências na Internet, como o Google ${ }^{\mathrm{TM}}$, Google Acadêmico ${ }^{\mathrm{TM}}$, Scielo e Portal de periódicos da CAPES. 


\section{Resultados e Discussão}

\subsection{O ecossistema manguezal eu sua presença no Brasil e no mundo}

Os manguezais são ecossistemas presentes em margens de baías, enseadas, foz de rios, lagunas e reentrâncias costeiras, na local de junção das águas dos rios com a do mar. São sistemas complexos, extremamente resistentes e resilientes, por isto consequentemente estáveis. Estão sempre influenciados pela ação diária das marés de água salgada ou salobra (Schaeffer-Novelli, 1999). Segundo Kathiresan e Binghan (2001), o mangue, como é denominado o grupo de plantas superiores presentes no ecossistema, é provavelmente o grupo mais desenvolvido morfológica, biológica, ecológica e fisiologicamente entre todos os existentes, por se desenvolverem satisfatoriamente em condições extremas, nas quais outras espécies não sobreviveriam.

Esta feição ecossistêmica está distribuída mundialmente nas regiões intertropicais, limitados às latitudes $30^{\circ} \mathrm{N}$ e $30^{\circ}$ $\mathrm{S}$, com exceção de sua presença mais ao norte em Bermuda, nos $32^{\circ} \mathrm{N}$, e no sul da Austrália, nos $38^{\circ} \mathrm{S}$ (Figura 1 ). Ocupam aproximadamente $136 \mathrm{mil} \mathrm{km²}$ em todo o mundo, distribuídos principalmente no Sul, Oeste e no Sudeste da Ásia, que apresentam 38,53\% da presença deste ecossistema (Tabela 1) (Spalding \& Leal, 2021).

Figura 1 - Distribuição dos manguezais na faixa intertropicais e suas exceções.

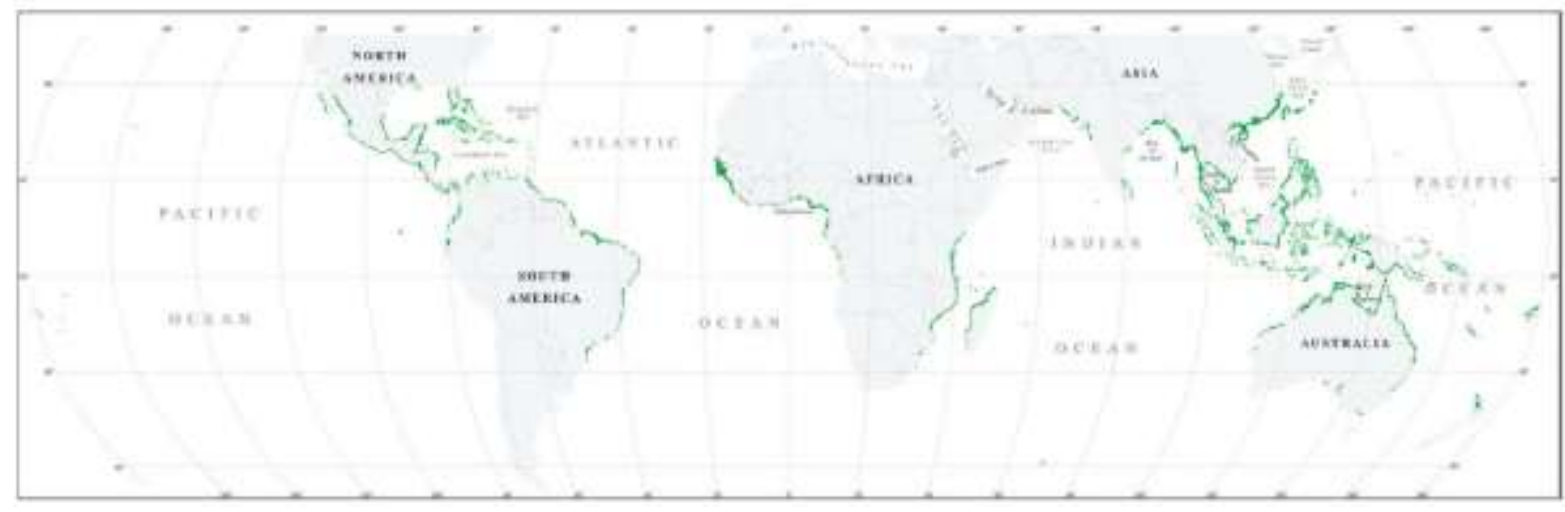

Fonte: Giri et al. (2011).

Tabela 1 - Área de cobertura das Florestas de Mangue no Mundo.

\begin{tabular}{l|c}
\multicolumn{1}{c|}{ Região do mundo } & Área de Manguezais $\left.\mathbf{( k m}^{\mathbf{2}}\right)$ \\
\hline Sul, Oeste e Sudeste da Ásia & 52.352 \\
\hline Américas e Caribe & 39.905 \\
\hline África Central e Oeste & 19.767 \\
\hline Austrália e Nova Zelândia & 9.983 \\
\hline Leste e Sul da África & 7.276 \\
\hline Ilhas do Pacífico & 6.285 \\
\hline Oriente Médio & 315 \\
\hline Total & $\mathbf{1 3 5 , 8 8 3}$
\end{tabular}

Fonte: Elaboração própria a partir de Spalding e Leal (2021).

No Brasil, são encontrados desde $04^{\circ} 20^{\prime}$ N, iniciando-se no Parque Nacional do Cabo Orange se encerrando na Laguna Imaruí, aos 28³0’ S (ICMBio, 2018a). Ocupam uma área de 13.989 km², distribuídos em 123 unidades de conservação, entre áreas de Proteção integra (PI) e Uso Sustentável (US) (Tabela 2) (ICMBio, 2018b). 
Tabela 2 - Manguezais por estado e percentual em relação ao total de manguezais no Brasil.

\begin{tabular}{l|c|c}
\multicolumn{1}{c|}{ Estado } & Área $\left(\mathbf{K m}^{\mathbf{2}}\right)$ & Percentual da Área Total (\%) \\
\hline Maranhão & 5054,90 & 36,13 \\
\hline Pará & 3905,89 & 27,92 \\
\hline Amapá & 2268,94 & 16,22 \\
\hline Bahia & 889,32 & 6,36 \\
\hline Paraná & 311,99 & 2,23 \\
\hline Sergipe & 265,43 & 1,90 \\
\hline São Paulo & 222,87 & 1,59 \\
\hline Ceará & 195,18 & 1,40 \\
\hline Pernambuco & 171,72 & 1,23 \\
\hline Rio de Janeiro & 137,79 & 0,98 \\
\hline Rio Grande do Norte & 135,35 & 0,97 \\
\hline Paraíba & 125,65 & 0,90 \\
\hline Santa Catarina & 104,01 & 0,74 \\
\hline Espírito Santo & 79,71 & 0,57 \\
\hline Piauí & 55,49 & 0,40 \\
\hline Alagoas & 55,35 & 0,40 \\
\hline Total & $\mathbf{1 3 . 9 8 9}$ & $\mathbf{1 0 0}$ \\
& \multicolumn{2}{|c}{ Fonte: Elaboração própria a partir de ICMBio $(2018 b)}$.
\end{tabular}

Apesar da grande abrangência, 13\% das áreas de manguezais ainda se encontram fora das Unidades de Conservação. Estes locais, por estarem nesta situação, possuem maior potencial de serem explorados sem nenhum tipo de controle por estarem fora do alcance dos órgãos responsáveis (Tabela 3).

Tabela 3 - Áreas de manguezal em Unidades de Conservação (federais, estaduais e municipais), nos anos de 2010 e 2014.

\begin{tabular}{l|c|c} 
Área de Manguezal & $\mathbf{2 0 1 0}$ & $\mathbf{2 0 1 4}$ \\
\hline UC de Proteção Integral (PI) & 1606,48 & 1998,91 \\
\hline UC de Uso Sustentável (US) & 7590,49 & 10115,53 \\
\hline Total PI + US & 9196,97 & 12114,44 \\
\hline \% total em UC & $\mathbf{7 5 \%}$ & $\mathbf{8 7 \%}$
\end{tabular}

Fonte: Elaboração própria a partir de ICMBio (2018b).

Mesmo apresentando números expressivos, os manguezais brasileiros sofrem com a sua exploração, através da extração de suas riquezas de forma indiscriminada. $\mathrm{O}$ desmatamento dos manguezais é uma das atividades de exploração mais antigas realizada no Brasil, remontando do século XVI, onde as árvores eram utilizadas para a extração do tanino, pigmento bastante utilizado à época para o tingimento de roupas (Vannucci, 2003). Atualmente, sabe-se que na costa brasileira 360 km² de manguezais foram convertidos em de criadouros de camarão, entre os anos de 2013 e 2016. Além disto, as terras são utilizadas para agricultura, onde há intensa extração de madeira, pesca desordenada, instalação de indústrias e turismo irregular. De 1980 aos dias hoje, estima-se que o Brasil tenha perdido $10 \mathrm{~km}^{2}$, sobretudo nas áreas costeiras das regiões Sul e Sudeste. (ICMBio, 2018b). 


\subsection{Breve histórico de leis.}

Como marco da legislação, em 1965 foi sancionada a lei federal 4.771, atualizando o Código Florestal Brasileiro, datado incialmente de 1934. Esta lei foi o primeiro passo no reconhecimento ecológico da importância do ecossistema manguezal no Brasil, ao indicar no texto da lei que este é importante "em toda sua extensão". Em 1985, através da resolução 004 do Conselho Nacional do Meio Ambiente (CONAMA), foi finalmente reconhecido como "Reserva Ecológica" (Cassiano, 2016).

Houve um espaço considerável de tempo entre essas leis, onde o conceito da necessidade de sua conservação foi inserido. A primeira tinha como foco apenas fazer uma delimitação das terras em função da exploração econômica, enquanto a segunda foi um passo importante para o entendimento da importância dos ecossistemas na a manutenção da biodiversidade. A lei de 1934 não tinha como intenção trazer ecossistemas, como o manguezal, para o cerne da concepção de preservação, que só teria força anos depois. Já o Código Florestal, após atualizado, objetivava resguardar o mangue e suas associações, traz na letra da lei a ideia primordial para conduzir a futura necessidade da manutenção sustentável desses ecossistemas.

Nos Estados Unidos, especificamente no estado da Flórida, há o exemplo de como as leis trazem a necessidade da preservação dos manguezais em sua letra. A normativa 403.9323 do estado americano presente em "Mangrove Trimming and Preservation Act" de 1996 diz que "é intenção do Legislativo proteger e preservar os recursos valiosos dos manguezais para o nosso meio ambiente e economia da remoção não regulamentada, desfolhamento e destruição", e que "são propriedade pública ou em terras reservadas para conservação e preservação, ou mitigação, exceto quando necessário para proteger o público saúde, segurança e bem-estar, ou para melhorar o uso público de, ou o acesso a, áreas de conservação em de acordo com os planos de manejo aprovados".

Dessa forma o uso sustentável do manguezal, aqui tratado traz em seu entendimento, traz o conceito da exploração consciente, em especial por aqueles que residem próximos a estas regiões, que o envergam de diferentes formas. Diversas comunidades que antes realizavam o uso não-intensivo dos manguezais estão experimentando um aumento desta utilização, como reflexo da modernização das economias. Desta forma, criou-se a necessidade do estabelecimento de sua valoração, pois a subestimação disto em conjunto aos impactos destas atividades humanas são os principais fatores que contribuem para a expansão das perdas e degradações dos manguezais (Costa, 2017).

\subsection{Valoração dos manguezais}

Valorar ecossistemas é necessário para a formulação e elaboração de políticas públicas necessárias, desenvolvimento sustentável e manutenção dos recursos ambientais. Tem como função avaliar o valor econômico de um recurso ambiental através da determinação de sua equivalência, dos quais o homem estaria disposto a abrir mão por uma melhora em sua qualidade de vida (Carvalho et al., 2018). Bann (1998) diz que há empenho dos economistas em estabelecer a eficiência econômica dos manguezais como principal critério para o estabelecimento de investimento público e de políticas públicas para sua manutenção.

Esta valoração econômica ampara a capacidade que o manguezal possui em ser utilizado de forma sustentável. No entanto, a principal dificuldade na realização destas ações está em inicializar a ideia naqueles que estão diretamente ligados à sua exploração. O proveito exacerbado, sem que se haja a conscientização necessária alarma e demonstra a urgência de ações que regulem e coíbam a sua exploração, bem como sensibilizem a sociedade, principalmente no tocante àqueles que vivem deste, para a sua importância.

Em relação os manguezais, os seus valores estão diretamente ligados aos serviços que fornecem a sociedade e, principalmente, aos seres que fazem parte dos seus ecossistemas e que dele se sustentam. Por possuírem uma intensa entrada e saída de energia nos seus sistemas, resultando em uma alta produtividade, promovem um ambiente completo para a produção, reprodução e manutenção da vida. Indubitavelmente, por sua riqueza supracitada, os animais que dele fazem uso são altamente 
dependentes, onde se encontra a maior problemática relacionada a estes locais devido a sua alta exploração e destruição em todo o mundo. Dele, o homem também retira seu sustento através da pesca de diversas espécies de peixes e crustáceos, além da produção direta pelo meio da aquicultura. Fornecem locais onde é possível a realização de visitação, tornando-se centros de interesse turístico e trazendo desta forma grandes potenciais econômicos para as cidades onde se inserem.

\subsection{Ações de conservação realizadas no Brasil e no Mundo e a educação ambiental}

O intenso aumento das zonas urbanas nas últimas décadas é um dos principais motivos da perda das áreas de mangue em todo o mundo, percebendo-se principalmente em países em desenvolvimento. Isto vem interferindo criticamente em suas dinâmicas locais e, consequentemente, nos seus serviços ecossistêmicos (Blotta et al, 2021).

A convenção de Ramsar, segundo o Ministério do Meio Ambiente (MMA), é um tratado intergovernamental que estabelece que as zonas úmidas dos países signatários devem ser utilizadas de forma sustentável, ou seja, para que todas as gerações atuais e posteriores possuam a oportunidade de utilizar seus recursos. Atualmente, o país possui $20 \%$ da biodiversidade da Terra, ocupando o $4^{\circ}$ lugar em superfície na Lista Ramsar. Junto ao Brasil, 133 países também participam da convenção, com 1079 sítios. O Brasil assinou a convenção de Ramsar em 02/02/1971, sendo aprovada pelo decreto n$^{\circ} 33$ de 16/06/1992.

Segundo Granziera, Adame e Gallo (2006), apesar de possuir sítios de extrema importância em seu território, o Brasil não tem em seu Direito mecanismos específicos que visem proteger estas áreas que sejam fundamentados na convenção Ramsar, sendo estas resguardadas pela Lei do Sistema Nacional de Unidades de Conservação (SNUC). Desta forma, o que existe é uma correspondência entre os sítios Ramsar brasileiros e as Unidades de Conservação (UC) nas áreas úmidas do país.

Além disto, a convenção trata como zonas úmidas as áreas de pântano, charco, turfa, ou água natural ou artificial, permanente ou temporária, doce, salobra ou salgada, incluindo as áreas de água marítima com menos de seis metros de profundidade em maré baixa. Aves aquáticas são pássaros dependentes destas zonas.

Como observado, no Brasil não existem ferramentas institucionais e jurídicas com a intenção direta de se trabalhar especificamente os manguezais em conjunto à sustentabilidade, dentro de um mesmo panorama. A convenção de Ramsar trata das áreas úmidas incluindo outras paisagens além dos manguezais. Os programas que estão inseridos dentro das UCs com objetivos do uso sustentável do mangue são realizados por projetos de ONGs e outras instituições que visam aliar educação ambiental com a conservação do ecossistema manguezal. No Brasil, estas ações são desenvolvidas visando o uso e a conservação destes locais, tomando como base os serviços ambientais como um todo prestados por estes à sociedade.

E, para que haja confluência entre sustentabilidade e a economia, elas devem ter como objetivo principal trazer os principais atores que, no caso, são aqueles que fazem das áreas de mangue sua principal fonte de renda. Segundo o ICMBio (2018b) 32 mil famílias retiram seu sustento dos manguezais somente em reservas federais, o que corrobora a importância destes para a esta população.

No Rio de Janeiro, o projeto Uçá da ONG Guardiões do Mar é um exemplo ao realizar a manutenção e o monitoramento das áreas de manguezais, além do fomento da educação ambiental e produção de conhecimento científico, colocando como atores os povos tradicionais, em unidades de conservação. Suas atividades desde 2012 conseguiram preservar 7 ha de mangue e plantar 64 mil mudas em 8 municípios do Rio de Janeiro. Também são responsáveis pela retirada de dezenas de toneladas de resíduos sólidos nos manguezais (Figura 2). 
Figura 2 - Lixo retirado de um manguezal pelo projeto UÇÁ.

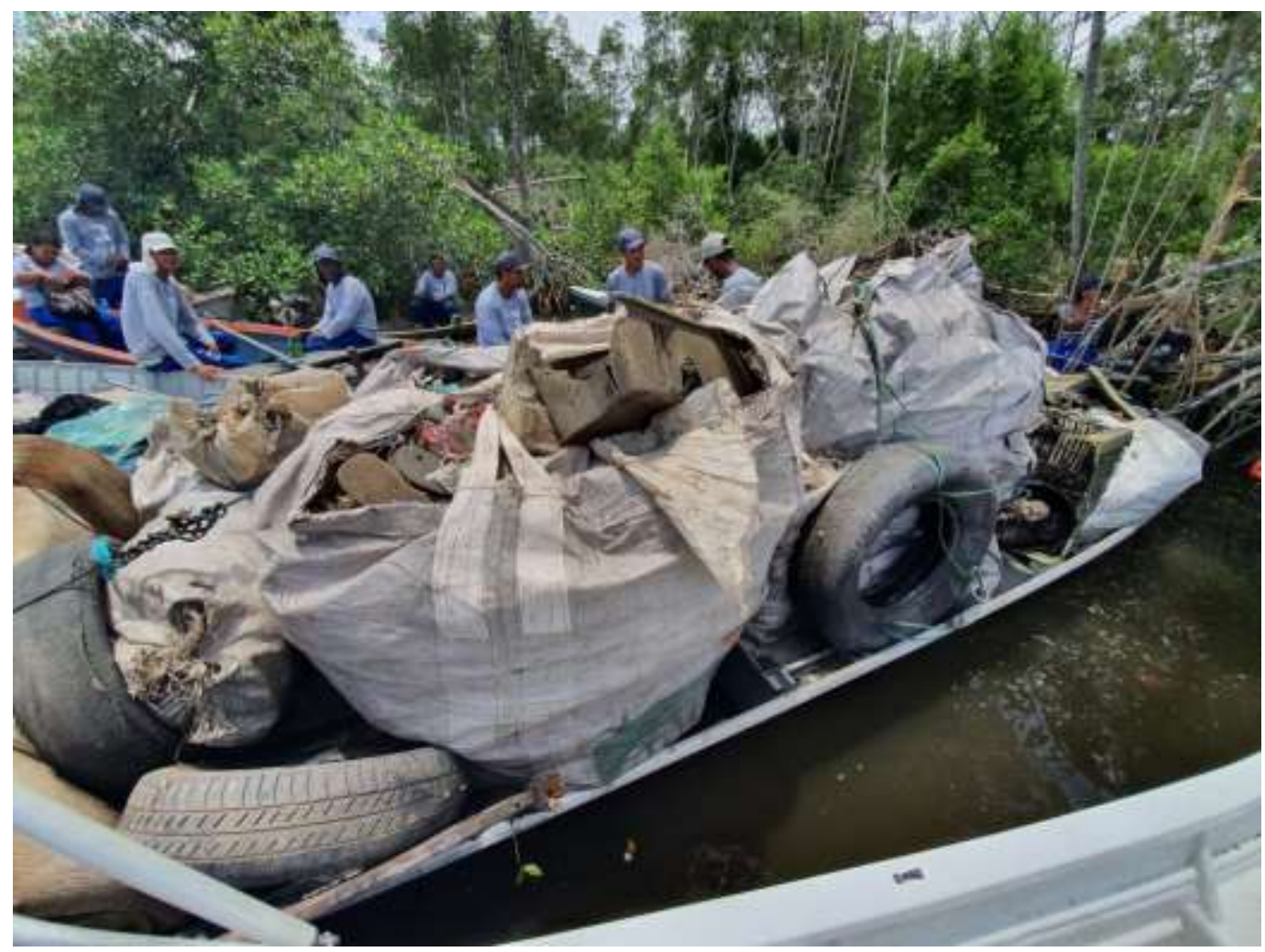

Fonte: Retirado de: http://projetouca.org.br/2021/09/catadores-e-pescadores-artesanais-limpam-a-baia-de-guanabara-durante-o-defeso/

No estado do Pará há também como iniciativa o projeto Mangues da Amazônia, sendo resultado da parceria entre Instituto Peabiru, Laboratório de Ecologia de Manguezal (LAMA), da Universidade Federal do Pará (UFPA), e a Associação Sarambuí, patrocinados pela Petrobras através do Programa Petrobras Socioambiental. Este projeto ocorrerá durante o ano de 2021, e terá como objetivo central agir na restauração do ecossistema manguezal, além de promover ações de capacitação e educação ambiental que beneficiará mais de 1600 pessoas nos municípios de Augusto Corrêa, Bragança e Tracuateua, no Pará.

Percebe-se que nos dois projetos supracitados, a educação ambiental é a base das ações realizadas. Ela age como uma ferramenta concreta e expressiva, na construção de um melhor relacionamento entre o homem e a natureza, inserindo-se de forma importante ao evidenciar o contexto histórico local, onde os resultados são emancipatórios, transformadores e únicos (Miranda et al, 2016).

Em outros países, projetos importantes merecem destaque no tocante a iniciativa de preservação dos manguezais em conjunto a sustentabilidade. A Pacific Mangroves Initiative (PMI) é um deles. Começou suas atividades em 2009 até meados de 2015, com a missão de implantar políticas de gestão nos manguezais nas ilhas do pacífico, onde os habitantes vivem principalmente da pesca e da coleta de recursos destas áreas. Duas atividades principais foram desenvolvidas pelo projeto, uma delas em Fiji, Samoa, Ilhas Salomão, Tonga e Vanuatu, com a intenção de melhorar os meios de subsistência e conservação da biodiversidade, bem como capacitar as comunidades a melhorar a gestão dos manguezais; a segunda tratou diretamente de ações em conjunto a universidades, instituições nacionais e a sociedade civil, para melhorar o conhecimento geral dos aspectos biológicos, econômicos, sociais e culturais dos recursos ambientais dos manguezais em cada país participante. 


\section{Conclusão}

As leis brasileiras que tratam os manguezais em sua letra ainda são incipientes ao desconsiderar as atividades necessárias para a sua preservação e em realizar uma especificação necessária que visaria dar uma importância ainda maior em sua conservação. Os sítios RAMSAR, apesar da extrema importância que possuem, generalizam a preservação de todas as áreas úmidas pelos países assinantes, onde o manguezal entra como uma delas. Isto faz com que as estas leis sigam um padrão igualmente generalista, desfocando a necessidade individual dos manguezais em receber leis e projetos específicos, como se observa na legislação brasileira.

A valoração do meio ambiente, principalmente dos manguezais alvos desse estudo, tem sua necessidade demonstrada ao se analisar que a ausência de valor monetário discreto faz com que o conhecimento da importância destas áreas seja renegado. No que tange a importância dos serviços ambientais, é extremamente relevante mensurar as suas riquezas, pois destas advêm a matéria-prima necessária para a subsistência da sociedade. Neste aspecto, pesquisas que tenham como finalidade estudar meios de preservação e de valoração dos serviços ambientais presentes nos ecossistemas manguezais são extremamente necessárias e devem ser incentivadas.

Fica clara, através dos exemplos aqui demonstrados, a importância da educação ambiental ao se analisar os projetos de preservação. Ao dar a importância devida às comunidades presentes na região, tem-se como recompensa a conscientização necessária dos principais povos atores presentes e que mais sofrem a influência direta das ações que degradam estes locais.

Por fim, os projetos apresentados demonstram indubitavelmente que ao se valorizar o manguezal e trabalhar em conjunto aos povos que dele tiram seu sustento, os resultados obtidos são extremamente importantes para que o uso sustentável e a gestão deste ecossistema sejam efetivos e atinjam resultados satisfatórios, além do benefício direto às populações em questões ambientais, sociais e econômicas.

\section{Referências}

Bann, C. (1998). The economic valuation of mangroves: A manual for researchers. Economy and Enviroment Program for Southeast Asia, 64.

Blotta, K. D., Guimarães, L. L., Braz, E. M. Q., Magenta, M. A. G., Ribeiro, R. B., \& Giordano, F. (2021). Diagnóstico de Manguezais Periurbanos após 20 anos de impactos antrópicos. Research, Society and Development, 10(1).

Carvalho, A. V. de, Bastos, M. de N. do C., Gurgel, E. S. C., Harada, A. Y., Nascimento, M. I. H. do, Marceliano, M. L. V., Silva, M. das G. S. da, \& Gomes, A. de L. S. (2018). Valoração econômica contingente dos recursos ambientais do ecossistema manguezal em São Caetano de Odivelas (PA). Revista IberoAmericana de Ciências Ambientais, 9(6), 409-427.

Cassiano, L. da A. (2016). Pagamento por serviços ambientais: Uma alternativa para a preservação das áreas de mangue do estuário do rio Paraíba. [Monografia]. Universidade Federal da Paraíba.

Costa, D. F. da S. (2017). Serviços ecossistêmicos prestados pelos manguezais do Rio Grande do Norte (nordeste do Brasil). Os desafios da Geografia Física na Fronteira do Conhecimento, 1127-1136. https://doi.org/10.20396/sbgfa.v1i2017.2025.

Florida State Law (1996). Mangrove Trimming \& Preservation Act. 9321-9333.

Giri, C., Ochieng, E., Tieszen, L. L., Zhu, Z., Singh, A., Loveland, T., Masek, J., \& Duke, N. (2011). Status and distribution of mangrove forests of the world using earth observation satellite data. Gloval Ecology and Biogeography, 154-159.

Granziera, M. L. M., Adame, A., \& Gallo, G. N. (2006). Direito ambiental internacional. Conservação dos espaços e da biodiversidade. Convenção RAMSAR. 15, 21. http://www.publicadireito.com.br/conpedi/manaus/arquivos/anais/manaus/direito_ambiental_maria_luiza_m_granziera_e_outros.pdf.

Instituto Chico Mendes de Conservação da Biodiversidade. (2018a). Atlas dos Manguezais do Brasil.

Instituto Chico Mendes de Conservação da Biodiversidade. (2018b). Brazilian mangrove atlas. Instituto Chico Mendes de Conservação da Biodiversidade.

Instituto Peabiru (2021, outubro 10). "Mangues da Amazônia” promove educação ambiental e recuperação de áreas na maior faixa de manguezal contínuo do mundo. Recuperado de https://peabiru.org.br/2021/03/04/mangues-da-amazonia-promove-educacao-ambiental-e-recuperacao-de-areas-na-maior-faixa-demanguezal-continuo-do-mundo/.

Internacional Union for Conservation of Nature (1996). Pacific Mangroves Initiative. https://www.iucn.org/regions/oceania/our-work/deploying-nature-basedsolutions/water-and-wetlands/completed-projects/pacific-mangroves-initiative. 
Research, Society and Development, v. 10, n. 15, e585101523345, 2021

(CC BY 4.0) | ISSN 2525-3409 | DOI: http://dx.doi.org/10.33448/rsd-v10i15.23345

Kathiresan, K., \& Bingham, B. L. (2001). Biology of Mangroves and Mangrove Ecosystems. Advances in Marine Biology, 40, 81-251.

Medeiros, S. R. M. de, Carvalho, R. G. de, \& Pimenta, M. R. C. (2014, dezembro). A proteção do ecossistema manguezal a luz da lei 12.651/2012: Novos desafios para a sustentabilidade dos manguezais do Rio Grande do Norte. Geotemas, 4(2), 59-78.

Ministério do Meio Ambiente (2021, outubro 1). Áreas Úmidas - Convenção de Ramsar. https://antigo.mma.gov.br/biodiversidade/biodiversidadeaquatica/zonas-umidas convencao-de-ramsar.html.

Miranda, A. M., Marinho, A. da S., Farias, C. S. S., \& Meireles, A. J. (2016). O valor do manguezal: Educação ambiental como instrumento social—Praia de Mundaú-Trairí/CE. Revista de Educação Ambiental, 21(2), 72-86.

Nova Mata (2021, novembro 4). Projeto Uçá. https://novamata.org/iniciativa/projeto-uca/.

Schaeffer-Novelli, Y. (1999) Grupo de Ecossistemas: Manguezal, Marisma e Apicum.

Spalding, M., \& Leal, M. (2021). The state of the world's mangroves 2021. Global Mangrove Alliance.

Vannucci, M. (2003). Os manguezais e nós: uma síntese de percepções. Edusp. 